\title{
The important challenge of quantifying tropical diversity
}

\author{
Anne E. Magurran
}

\begin{abstract}
The tropics are the repository of much of the world's biodiversity, yet are undersampled relative to temperate regions. To help fill this knowledge gap, a paper in BMC Biology explores diversity patterns in tropical African plants, as revealed by the RAINBIO database. The paper documents spatial variation in diversity and data coverage, but also highlights the challenges faced in quantifying diversity patterns using data collated from a range of sources including herbaria.

See research article: http://bmcbiol.biomedcentral. com/articles/10.1186/s12915-017-0356-8.
\end{abstract}

\section{Commentary}

The far-reaching impacts that our human species is having on the Earth's ecosystems have led scientists to call the present era the Anthropocene. There can be no doubt that the world's biodiversity is under unprecedented threat. Species extinctions make headline news, while natural communities are being reorganized at a rate that far exceeds historical baselines [1]. Yet, despite growing concern about the fate of the biosphere, substantial knowledge gaps with respect to the distribution and status of species remain. Most ecologists and taxonomists are based in temperate regions [2], which are also the most comprehensively surveyed. Large swathes of the Earth, particularly biodiverse tropical regions, are very poorly documented [3] (Fig. 1). A new paper, by Sosef et al. [4], reporting a synthesis of diversity patterns in tropical African plants [5], is thus an important and timely contribution.

The gold standard for biodiversity assessment is representative sampling using consistent and appropriate

Correspondence: aem1@st-andrews.ac.uk

Centre for Biological Diversity, School of Biology, University of St Andrews, St Andrews, Scotland, UK 


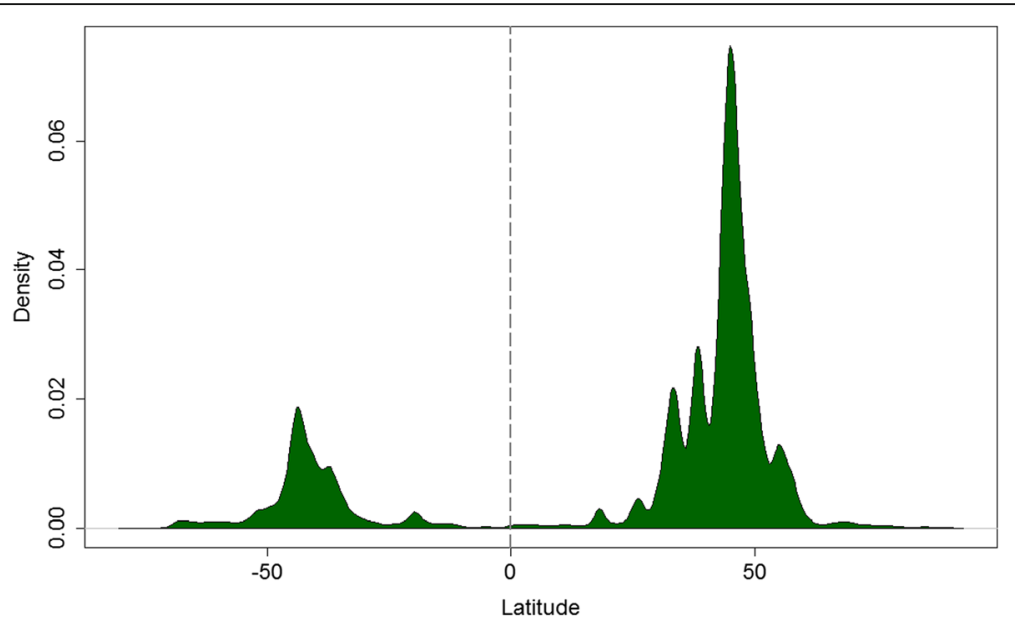

Fig. 1. Rigorous ecological survey data are strongly concentrated in temperate latitudes. For example, this plot illustrates the density of survey points, in relation to latitude (degrees north and south, with the equator indicated by the dashed line), in the BioTIME database of assemblage time series [1]. The dearth of these data from tropical regions demonstrates the importance of databases [5] and analyses, as in [4], which draw on different data sources

museums value breadth and rarity in their collections, databases populated from these sources are prone to overemphasizing singleton and doubleton species. However, it is worth noting that rigorous surveys of tropical ecosystems similarly report high numbers of singleton taxa [9].

Other metrics employed in the paper, such as the Neilsen diversity metric, are less sensitive to sampling effort than richness estimators. Nonetheless, it is important to remember that the value the Neilsen statistic calculates-dubbed 'effective species'-refers to an attribute of the data set and does not necessarily provide an ecologically meaningful insight into the structure of the assemblages from which the data were sourced. Even turnover metrics, which evaluate compositional change over space or time and might seem to be less obviously affected by sampling effort, can be influenced by inconsistencies in sampling [8].

These caveats could suggest that analyses based on heterogeneous databases are so fraught with problems as to be uninformative. Yet, provided that the results are interpreted cautiously, such analyses can provide a crucial initial step towards a better understanding of diversity patterns and help steer future investigations towards productive quests. The first contribution of these analyses is that they make plain the extent of heterogeneity in data collection over space and time. Although collecting dates in RAINBIO range from 1782 to 2015, collecting intensity is not constant. For instance, recording in the Democratic Republic of Congo occurred mostly from the 1930s to the 1960s [4]. Clear recommendations for improving understanding of tropical diversity, such as improved data exchange between datasets, are identified.

A second contribution of analyses of databases such as RAINBIO is that firmer estimates of richness, endemicity, range extent and patterns of commonness and rarity begin to emerge. While is it wise to be careful about estimates of richness and reported values of diversity statistics, especially when making comparisons with other regions and systems, these results help shape hypotheses and guide objectives for future studies. For example, Sosef and colleagues' [4] analysis of diversity patterns and data coverage is well placed to inform new investigations on a range of topics, such as the consequences for biodiversity of climate change, and to highlight the groups and regions in which taxonomic research is most urgently needed.

New insights into functional diversity represent a third contribution of an analysis such as this [4]. Sosef et al. report geographic variation in plant growth form dominance across tropical Africa, a field of research in which data have previously been scarce. This advance is possible because the observed diversity patterns are underpinned by georeferenced data on named species.

Finally, a macroecological analysis such as Sosef et al.'s, can be viewed alongside other investigations of data with a similar geographical scope, for example Marshall et al.'s [10] recent assessment of rarity amongst the tropical African flora. Of course different compilations of data may share many of the same shortcomings, not least because some overlap in source material is likely, but as long as they are not interpreted too simplistically, complementary analyses such as Sosef et al.'s [4] and Marshall et al.'s [10] jointly advance understanding and are well placed to support the conservation of biodiversity in our rapidly changing world. In particular they underline the pressing need for better understanding of the distribution and nature of tropical diversity. Innovative and insightful analyses of data in RAINBIO and other databases will be key to achieving this aim. 


\section{Acknowledgements}

I thank Faye Moyes for constructing Fig. 1.

\section{Funding}

AEM is supported from ERC grants BioTIME (250189) and BioCHANGE (727440).

\section{Authors' contributions}

AEM wrote this article.

\section{Competing interests}

The author declares that she has no competing interests.

Published online: 07 March 2017

\section{References}

1. Dornelas M, Gotelli NJ, McGill BJ, Shimadzu H, Moyes F, Sievers C, et al. Assemblage time series reveal biodiversity change but not systematic loss. Science. 2014;344:296-9.

2. Gaston KJ, May RM. The taxonomy of taxonomists. Nature. 1992;356:281-2.

3. Engemann K, Enquist BJ, Sandel B, Boyle B, Jørgensen PM, Morueta-Holme $\mathrm{N}$, et al. Limited sampling hampers "big data" estimation of species richness in a tropical biodiversity hotspot. Ecol Evol. 2015;5(3):807-20.

4. Sosef MSM, et al. Exploring the floristic diversity of tropical Africa. BMC Biol. 2017. http://bmcbiol.biomedcentral.com/articles/10.1186/s12915-017-0356-8.

5. Gilles D, Zaiss R, Blach-Overgaard A. Catarino Ls, Damen T, Deblauwe V, et al. RAINBIO: a mega-database of tropical African vascular plants distributions. PhytoKeys. 2016;74:1-18.

6. Buckland ST, Baillie SR, Dick JM, Elston DA, Magurran AE, Scott EM, et al. How should regional biodiversity be monitored? Environmental Ecologica Stat. 2012;19(4):601-26

7. Colwell RK, Coddington JA. Estimating terrestrial biodiversity through extrapolation. Phil Trans R Soc Lond B. 1994;345:101-18.

8. Magurran AE. Measuring biological diversity. Oxford: Blackwell Science; 2004

9. Coddington JA, Agnarsson I, Miller JA, Kuntner M, Hormiga G. Undersampling bias: the null hypothesis for singleton species in tropical arthropod surveys. J Animal Ecol. 2009:78:573-84.

10. Marshall CAM, Wieringa JJ, Hawthorne WD. Bioquality hotspots in the tropical African flora. Curr Biol. 2016:26(23):3214-9. 